Muséologies

Les cahiers d'études supérieures

muséologies

\title{
Geneviève Chevalier, artiste-commissaire ou l'art de concevoir une exposition
}

\section{Chantal Steegmuller}

Volume 4, numéro 2, printemps 2010

URI : https://id.erudit.org/iderudit/1033543ar

DOI : https://doi.org/10.7202/1033543ar

Aller au sommaire du numéro

Éditeur(s)

Association Québécoise de Promotion des Recherches Étudiantes en

Muséologie (AQPREM)

ISSN

1718-5181 (imprimé)

1929-7815 (numérique)

Découvrir la revue

Citer ce compte rendu

Steegmuller, C. (2010). Compte rendu de [Geneviève Chevalier,

artiste-commissaire ou l'art de concevoir une exposition]. Muséologies, 4(2),

132-139. https://doi.org/10.7202/1033543ar d'utilisation que vous pouvez consulter en ligne.

https://apropos.erudit.org/fr/usagers/politique-dutilisation/ 


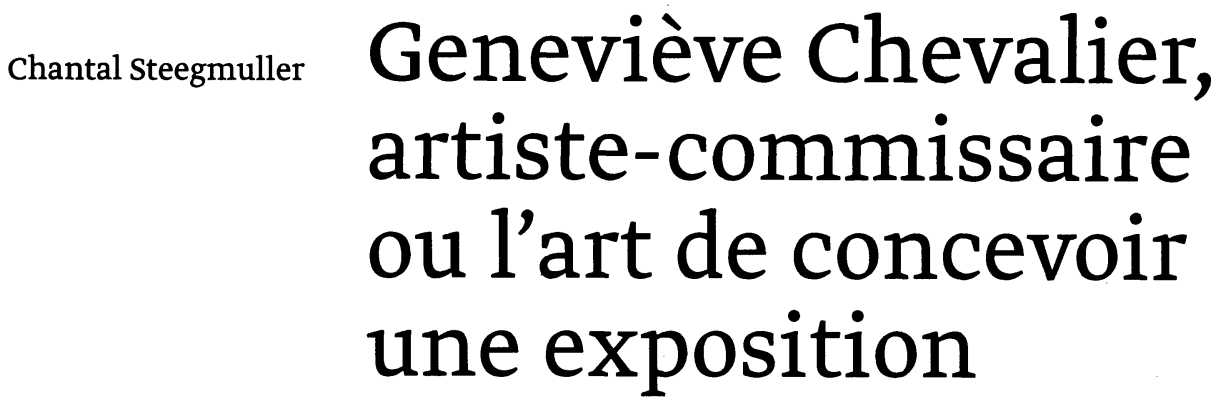

Ceneviève Chevalier est une artiste en arts visuels qui vit et travaille dans les Cantons de I'Est. Elle détient une maîtrise en beaux-arts, spécialisation sculpture, de l'Université Concordia, ainsi qu'un baccalauréat en arts plastiques de l'Université Laval. Co-conservatrice à la Galerie d'art Foreman de l'Université Bishop's, elle est présentement étudiante au doctorat en étude et pratique des arts à l'Université du Québec à Montréal, sous la direction de Marie Fraser. 


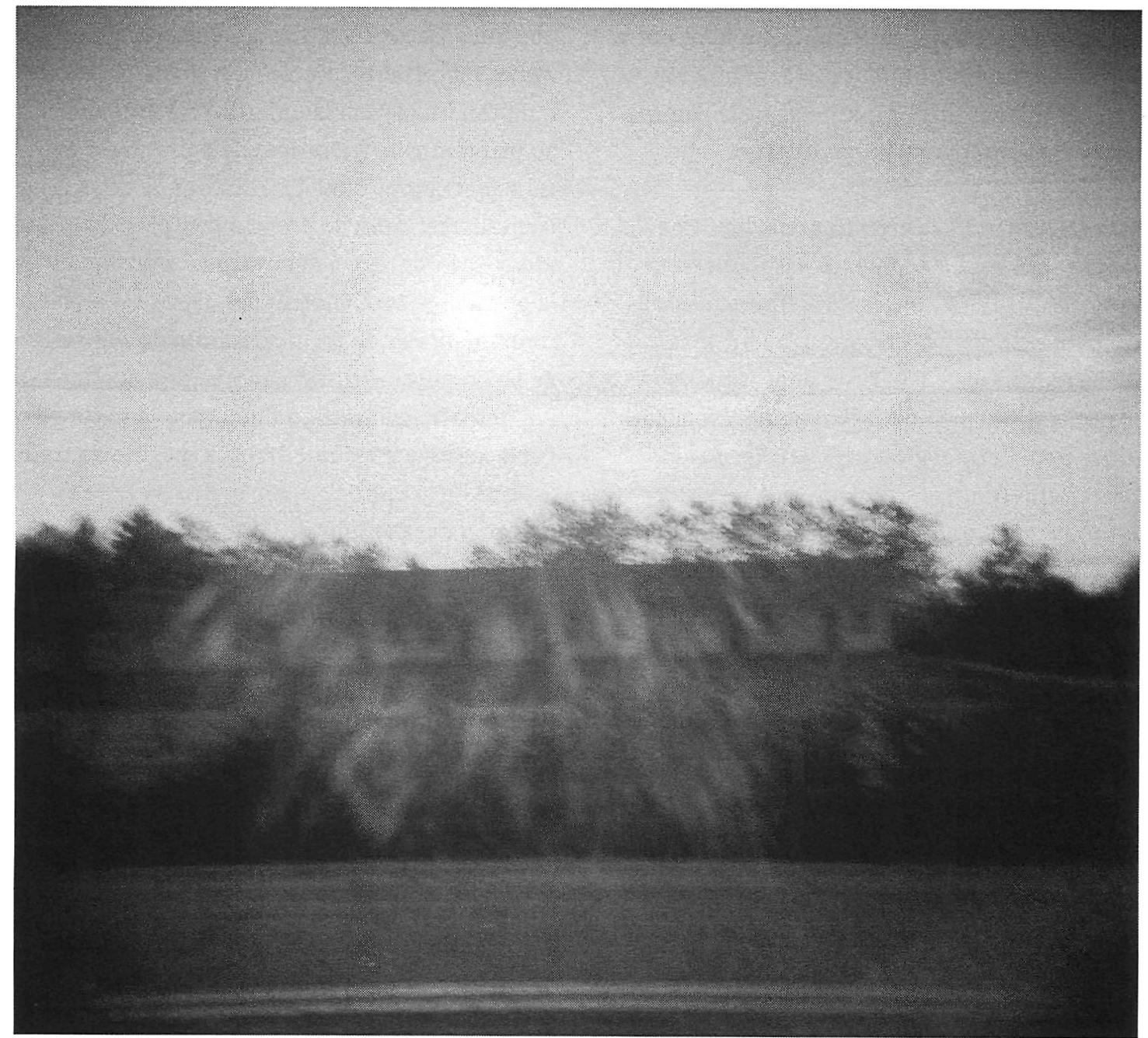


Artiste ou commissaire, Geneviève Chevalier s'amuse à changer de rôle au gré de ses projets et à questionner les implications de chacun, à travers sa double pratique. Dans ses plus récentes œuvres, qu'il s'agisse d'installations, de sculptures, de dessins, de photographies ou de projets Web, l'artiste s'intéresse aux questions liées à loccupation du paysage et à la façon dont notre héritage culturel influe sur cette occupation. Ce sont ces mêmes préoccupations que la commissaire développe dans ses expositions.

Pour sa recherche doctorale, Geneviève Chevalier s'intéresse au contexte particulier des Cantons de l'Est. Le choix de son sujet d'étude s'explique de diverses façons. D'abord, le fait que la Galerie d'art Foreman, pour laquelle elle travaille depuis maintenant deux ans, soit située dans cette région, plus précisément à Lennoxville, motive sa volonté de présenter des projets qui s'adressent spécifiquement aux gens de la communauté. Convier des artistes à se pencher sur des sujets propres au lieu où sont exposées les œuvres est ainsi, pour elle, un point crucial. C'est l'occasion de donner une voix, par exemple, à la ruralité, une réalité notable du Québec, qui s'éloigne de la métropole et de son urbanité. Aussi, le fait de vivre dans cette région lui permet-il d'être pleinement consciente de l'importance de certains enjeux locaux, tant géographiques qu'historiques ou politiques, lui donnant de ce fait la possibilité de susciter une réflexion critique.
C'est ainsi des particularités de cette région qu'elle s'inspirera pour concevoir une série de trois expositions. Motelisation, qui sera présentée à la Galerie d'art Foreman de l'Université Bishop's, du 21 avril au 19 juin 2010, abordera l'architecture des motels, mais surtout le glissement qui s'est effectué dans la perception que nous en avons, d'hier à aujourd'hui. Le Projet Stanstead ou comment traverser la frontière, qui prendra place à la Galerie d'art Foreman au printemps 2011, traitera de politique et des transformations dans les mesures de sécurité frontalières. Enfin, le projet sur le parc du Mont-Orford (titre à déterminer), prévu pour le printemps 2012, traitera des questions environnementales de la privatisation du parc et, de façon plus large, des parcs nationaux dans l'imaginaire commun, à l'heure où la nature à l'état sauvage disparaît! Trois expositions, trois aspects de la culture locale et de l'histoire de la région des Cantons de l'Est.

\section{Motelisation $^{1}$}

Pour la commissaire-artiste, la conception de Motelisation a débuté par une recherche sur les motels nord-américains. L'intérêt de mettre en place une telle exposition vient du fait que, pour Geneviève Chevalier, les motels se veulent " caractéristiques " tant d'une culture et d'un contexte historique singuliers que de Lennoxville, où est installée la Galerie d'art 
Foreman. En effet, la petite ville compte quatre motels, tous situés sur la rue Queen. La Galerie entretient d'ailleurs un lien particulier avec ces établissements, puisque c'est là que logent tous les artistes qui y exposent.

La commissaire a donc convié six artistes ${ }^{2}$, choisis en raison de leur approche et de leur pratique artistiques, à réfléchir à cette thématique et à explorer diverses questions en lien avec ces lieux spécifiques. Qu'est-ce que le motel en réalité? Dans quel contexte a-t-il émergé ? Que signifie sa prolifération et qu'est-ce qui a provoqué son déclin?

Andrée Anne Vien adopte une approche relationnelle/contextuelle, combinant des aspects propres à l'anthropologie et à la sociologie, en s'intéressant à l'expérience vécue par les clients du Motel Lennoxville. L'œuvre Note de Motel est accrochée, tels des rideaux brodés d'extraits de récits de voyageurs, à la fenêtre de toutes les chambres de l'établissement. L'artiste compte également " parasiter" le site Web du motel, en permettant une visite interactive des différentes pièces à louer.

Alison S.M. Kobayashi et Gintas Tirilis créent, pour leur part, une installation composée d'artefacts récoltés lors de leur passage dans divers

1

"Motelisation» est un mot inventé par Ceneviève Chevalier dans le cadre du projet d'exposition et qui évoque la prolifération des motels. motels désaffectés de la banlieue de Toronto, en 2009. Ces objets, témoins d'une époque maintenant révolue, soulignent le style vernaculaire du motel traditionnel, en voie de disparition, au profit des chaînes standardisées.

Chantal Séguin présente, quant à elle, une projection d'images numériques réalisées à partir de photographies prises dans les chambres du motel La Marquise, avec son décor éclectique et kitsch, auxquelles sont superposées des figures soigneusement tracées. Son œuvre aborde la fonction plus "cachée " de ces établissements, celle des rendez-vous plus ou moins galants! À travers sa pratique, l'artiste s'intéresse ainsi à l'architecture et au narratif propres aux relations humaines. Le romantisme qui caractérise habituellement ses œuvres est donc confronté à la réalité brute des activités de nature sexuelle qui se déroulent dans les motels.

Les œuvres présentées dans le cadre de l'exposition sont ainsi réalisées soit directement sur les sites des motels de Lennoxville, soit à partir de la thématique du motel.

Parallèlement à l'exposition Motelisation, Geneviève Chevalier, l'artiste, présente une œuvre réalisée pour l'Of Biennale du centre Dare-Dare, en avril

2

En plus des quatre artistes cités dans le présent carnet, Andrew King, architecte de formation et de pratique, et sa conjointe, Angela Silver, réaliseront également une œuvre pour l'exposition Motelisation. 

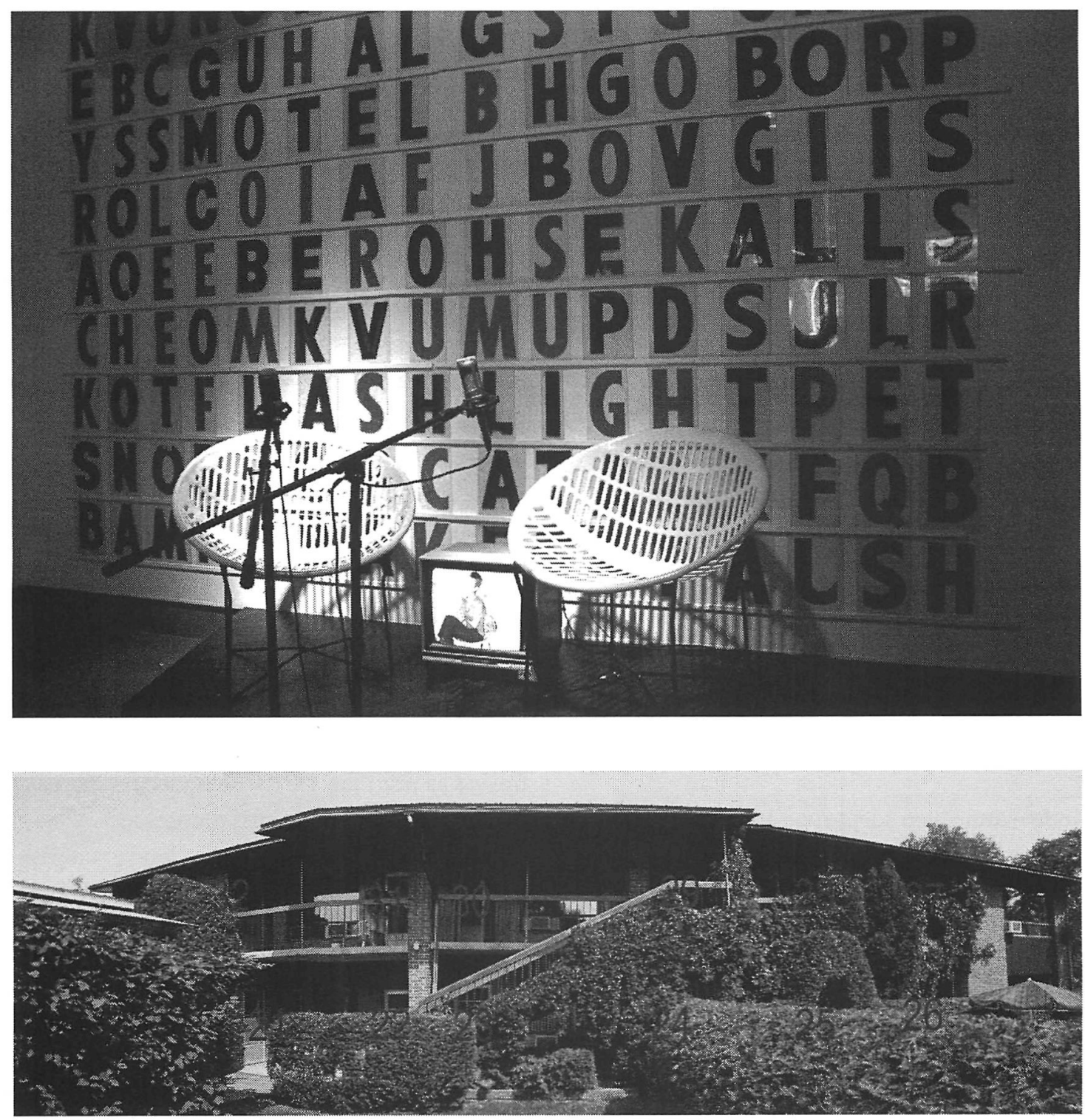

Illustration 1

"No Vacancy». Alison S.M. Kobayashi et Gintas Tirilis, installation, 2009.
Illustration 2

Site Web du Motel Lennoxville, $<$ http://www.lennoxville.qc.ca/motel/ index.html>. 


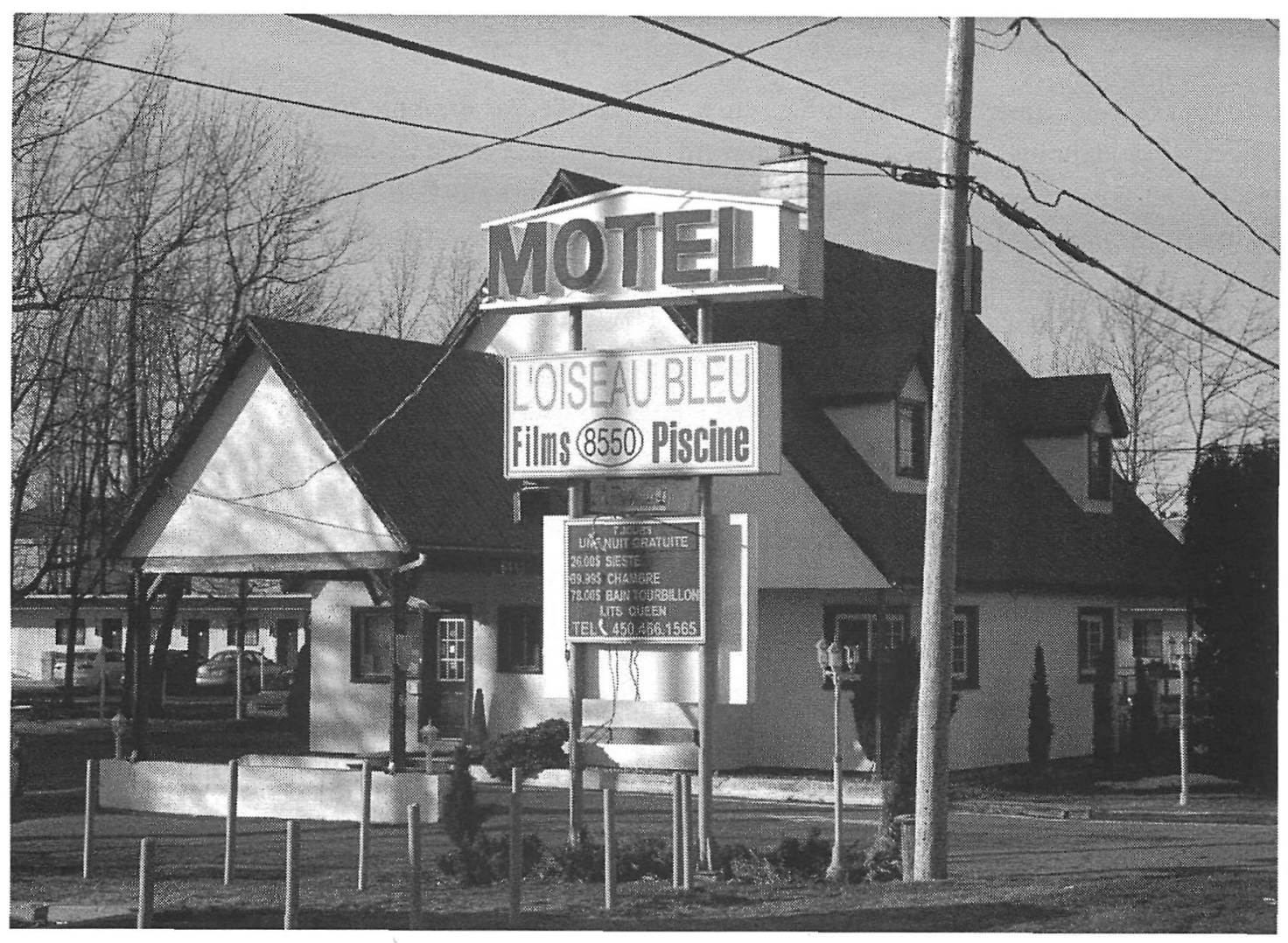

Illustration 3

Motel L'Oiseau Bleu, boulevard

Taschereau.

Photo: Geneviève Chevalier, 2010 
2010. Des fiches imprimées, disponibles chez Dare-Dare $^{3}$ et dans certains motels du boulevard Taschereau, permettent aux participants de (re) découvrir les motels et les sites d'intérêt, lors d'un parcours routier sur le boulevard. L'œuvre aborde la question de la perception que les gens ont des motels aujourd'hui et celle qu'on en avait autrefois. L'artiste souhaite, par cette visite " temporelle" du "avant-après ", permettre aux visiteurs-automobilistes d'entrevoir l'évolution de cette banlieue de la Rive-Sud de Montréal sous l'effet de l'urbanisme.

\section{Projet Stanstead ou comment traverser la frontière}

La seconde exposition, qui ouvrira ses portes au printemps 2011, s'intéressera à la ville de Stanstead, localisée précisément à la frontière entre le Canada et les États-Unis. La situation exceptionnelle de cette ville, où les habitants avaient l'habitude de circuler librement des deux côtés de la frontière, a récemment changé en raison de l'application de nouvelles mesures de sécurité renforcées : des barrières ont été installées pour séparer les deux pays. Le résultat est hors du commun, car la séparation remet en question jusqu'à l'identité même de

Les fiches seront également disponibles sur le site Internet du centre Dare-Dare : <http://www.dare-dare.org/en/ off-biennales. la ville. Par exemple, lorsqu'on va à la bibliothèque, on cherche les livres au Canada et on les emprunte aux États-Unis. La foule qui assiste à un spectacle est assise aux États-Unis alors que les comédiens jouent au Canada.

Le projet, en deux étapes, combinera à la fois des interventions artistiques in situ et une exposition à la Galerie d'art Foreman. C'est dans la salle de cette dernière, lieu de discussion et de réflexion, que sera présentée une série d'artefacts provenant de la collection de la Société historique de Stanstead et relatant l'histoire de la ville et de sa frontière canado-américaine. Les interventions réalisées in situ y seront également documentées. Geneviève Chevalier s'intéressera ainsi à toute la question de l'identité et de la réorganisation de la vie locale de Stanstead et, par extension, à la question des frontières dans le monde.

\section{Projet sur le parc du Mont-Orford}

La troisième et dernière exposition de la série sera présentée en 2012, en partenariat avec le Centre d'art Orford ${ }^{4}$. Afin de réfléchir plus largement à la question de l'environnement, Geneviève Chevalier invitera des artistes à

4

Le Centre d'art Orford accueillera Benedict Fullalove au cours de l'été 2011, lors d'une conférence et durant la période des randonnées pédestres. 
se pencher sur le sujet des parcs nationaux, notamment celui du parc du Mont-Orford.

Avec la participation de Benedict Fullalove, historien de l'art albertain - spécialiste de la représentation du paysage - et alpiniste d'expérience, la commissaire-artiste conviera les visiteurs à des randonnées pédestres dans la section retirée du parc. En juin 2006, le gouvernement provincial a en effet retiré une section de 469 hectares du parc national du Mont-Orford, sous prétexte d'une nécessité de construire des condos (lors de l'adoption sous bâillon du projet de loi 23 / Loi permettant d'assurer l'agrandissement du parc national du Mont-Orford, la préservation de la biodiversité de territoires limitrophes et le maintien des activités récréotouristiques). Même si la population souhaite à plus de $75 \%$ la réintégration des terres dans le parc, le statut de cette section est toujours en suspens. Les expéditions se feront donc à partir d'une carte topographique représentant cette portion du territoire qui est au cœur du litige et que Geneviève Chevalier réalisera à partir de coordonnées GPS 5 .

Selon Geneviève Chevalier, cette série de trois expositions ne se veut pas une "trilogie", mais davantage une réflexion en plusieurs étapes sur la façon d'aborder le contexte local, tout en

5

Les coordonnées seront récoltées lors de randonnées effectuées par Ceneviève Chevalier, en compagnie de guides membres de regroupements d'amateurs du parc, à l'été 2010. La carte sera réalisée grâce à la contribution de la coalition citoyenne SOS parc Orford ainsi que des Amis du parc Orford. explorant des pratiques expérimentales de commissariat. Pour la commissaire-artiste, l'exposition est un médium qui permet d'engager le visiteur dans un espace de réflexion. Pour l'artiste-commissaire, la création d'une œuvre, par exemple une installation, et la conception d'une exposition, relèvent de méthodologies très semblables. C'est une appropriation de l'espace.

Au-delà du résultat " visitable ", l'exposition est aussi l'occasion de s'intéresser à la relation toute particulière qui existe et se développe entre l'artiste et le commissaire. Ce n'est pas, selon la doctorante, une collaboration au sens propre, mais bien plus un échange sur un thème commun. Pour Geneviève Chevalier, l'exposition offre la possibilité d'explorer un contexte réel, par le biais des propositions faites par les artistes invités. C'est aussi une manière de créer des situations, de présenter des œuvres aux visiteurs, afin de leur faire voir la multitude de possibilités et de points de vue, d'initier un dialogue autour de thèmes fondamentaux et rassembleurs, comme le paysage bâti et naturel. 